\title{
LOCAL COMPACTNESS AND HEWITT REALCOMPACTIFICATIONS OF PRODUCTS
}

\author{
HARUTO OHTA
}

\begin{abstract}
In this note we prove McArthur's conjecture [0]: If card $X$ is nonmeasurable and if $v(X \times Y)=v X \times v Y$ holds for each space $Y$, then $X$ is locally compact. Consequently, we can completely characterize the class of all spaces $X$ such that for each space $Y, v(X \times Y)=v X \times v Y$ holds.
\end{abstract}

1. Introduction. All spaces considered in this note will be completely regular Hausdorff. For a space $X, v X$ denotes the Hewitt realcompactification of $X$, and the symbolism $v(X \times Y)=v X \times v Y$ means that $X \times Y$ is $C$-embedded in $v X \times v Y$. Following [6], let $\Re$ denote the class of all spaces $X$ such that for each space $Y, v(X \times Y)=v X \times v Y$ holds. It is known that a locally compact realcompact space of nonmeasurable cardinal is a member of $R$ and that every member of $\Re$ is realcompact (Comfort [1, Corollary 2.2], McArthur [6, Theorem 5.2]). In [6], McArthur conjectured that if card $X$ is nonmeasurable and $X$ is a member of $\mathcal{R}$, then $X$ is locally compact. The main purpose of this note is to establish his conjecture positively. More precisely, we can prove the following theorems. The implication $(\mathrm{a}) \rightarrow(\mathrm{b})$ of Theorem 1 was proved by Comfort [1].

THEOREM 1. For a space $X$ of nonmeasurable cardinal the following conditions are equivalent:

(a) $X$ is locally compact.

(b) $X \times Y$ is $C$-embedded in $X \times v Y$ for each space $Y$.

THEOREM 2. For a space $X$ of nonmeasurable cardinal the following conditions are equivalent:

(a) $X$ is locally pseudocompact.

(b) $X \times Y$ is $C$-embedded in $X \times v Y$ for each $k$-space $Y$.

We remark that, in Theorems 1 and 2, the assumption "card $X$ is nonmeasurable" is useful only for the implication $(a) \rightarrow(b)$. Combining these theorems with the results of Comfort and McArthur, quoted above, and Hušek [4, Theorem 3], we have the following theorems.

THEOREM 3. For a space $X$ the following conditions are equivalent:

Received by the editors April 26, 1977 and, in revised form, September 29, 1977. AMS (MOS) subject classifications (1970). Primary 54D60; Secondary 54D45, 54B10.

Key words and phrases. Hewitt realcompactification, realcompact space, locally compact space, locally pseudocompact space, $k$-space, product space, topological completion. 
(a) $X$ is locally compact, realcompact and card $X$ is nonmeasurable.

(b) $v(X \times Y)=v X \times v Y$ holds for each space $Y$.

THEOREM 4. For a space $X$ the following conditions are equivalent:

(a) $v X$ is locally compact and card $X$ is nonmeasurable.

(b) $v(X \times Y)=v X \times v Y$ holds for each $k$-space $Y$.

For the notions of locally pseudocompact spaces and $k$-spaces see [1]. For an ordinal $\alpha$, we denote by $W(\alpha)$ the set of all ordinals less than $\alpha$ topologized with order topology, and by $\omega_{0}$ the first infinite ordinal. Other terms can be found in [3].

\section{Proofs of theorems.}

Proof of Theorem 1. (a) $\rightarrow$ (b). This is the result of Comfort [1, Theorem 2.1]. (b) $\rightarrow$ (a). Suppose, on the contrary, that $X$ is not locally compact at $x_{0} \in X$. Let $\left\{G_{\lambda} \mid \lambda \in \Lambda\right\}$ be a neighborhood base at $x_{0}$ in $X$. Then, for each $\lambda \in \Lambda, \mathrm{cl}_{X} G_{\lambda}$ is not compact, and thus there exists a point $x_{\lambda} \in \mathrm{cl}_{\beta X} G_{\lambda} \cap$ $(\beta X-X)$, where $\beta X$ is the Stone-Cech compactification of $X$. For each $\lambda \in \Lambda$, let $\left\{G(\lambda, \sigma) \mid \sigma \in \Sigma_{\lambda}\right\}$ be a neighborhood base at $x_{\lambda}$ in $\beta X$. For each $\sigma \in \Sigma_{\lambda}$, we can choose a point $x(\lambda, \sigma) \in X$ and an open set $H(\lambda, \sigma)$ in $X$ such that $x(\lambda, \sigma) \in H(\lambda, \sigma) \subset G(\lambda, \sigma) \cap G_{\lambda}$. Let $s_{\lambda}$ be an ideal point, and set $S_{\lambda}=\Sigma_{\lambda} \cup\left\{s_{\lambda}\right\}$, topologized as follows: Each point of $\Sigma_{\lambda}$ is isolated and $\left\{J(\lambda, \sigma) \mid \sigma \in \Sigma_{\lambda}\right\}$ is a neighborhood base at $s_{\lambda}$, where $J(\lambda, \sigma)=\left\{s_{\lambda}\right\} \cup\{\tau \in$ $\left.\Sigma_{\lambda} \mid G(\lambda, \sigma) \supset G(\lambda, \tau)\right\}$. Let $\mathfrak{n}$ be a regular cardinal greater than $\sup \left\{\right.$ card $\Sigma_{\lambda} \mid \lambda$ $\in \Lambda$, and let $\omega_{\alpha}$ be the initial ordinal of $n$. For each $\lambda \in \Lambda$, let

$$
T_{\lambda(1)}=\left\{(\lambda(1), \gamma, \beta) \mid \gamma \leqslant \omega_{\alpha}, \beta \leqslant \omega_{0}\right\}
$$

be the copy of $W\left(\omega_{\alpha}+1\right) \times W\left(\omega_{0}+1\right)$, and let

$$
T_{\lambda(2)}=\left\{(\lambda(2), \gamma, s) \mid \gamma \leqslant \omega_{\alpha}, s \in S_{\lambda}\right\}
$$

be the copy of $W\left(\omega_{\alpha}+1\right) \times S_{\lambda}$. By identifying a point $\left(\lambda(1), \gamma, \omega_{0}\right)$ with $\left(\lambda(2), \gamma, s_{\lambda}\right)$ for $\gamma \leqslant \omega_{\alpha}$, we have a quotient space $T_{\lambda}$ and a quotient map $f_{\lambda}$ : $T_{\lambda(1)} \oplus T_{\lambda(2)} \rightarrow T_{\lambda}$, where $A \oplus B$ denotes the topological sum of $A$ and $B$. Let us set $Z=\bigoplus\left\{T_{\lambda} \mid \lambda \in \Lambda\right\}$, and let $Y_{0}$ be the quotient space obtained from $Z$ be collapsing a set $\left\{f_{\lambda}\left(\left(\lambda(1), \omega_{\alpha}, \beta\right)\right) \mid \lambda \in \Lambda\right\}$ to a single point $y(\beta) \in Y_{0}$ for $\beta \leqslant \omega_{0}$. Let $g: Z \rightarrow Y_{0}$ be the quotient map, and set $h_{\lambda}=g \circ f_{\lambda}$ for each $\lambda \in \Lambda$. Then $y\left(\omega_{0}\right)=h_{\lambda}\left(\left(\lambda(2), \omega_{\alpha}, s_{\lambda}\right)\right)$ for each $\lambda \in \Lambda$. Let us set $Y=Y_{0}-$ $\left\{y_{0}\right\}$, where $y_{0}=y\left(\omega_{0}\right)$. We shall now prove that $Y_{0} \subset v Y$ by showing that $Y$ is $C$-embedded in $Y_{0}$. Let $\phi$ be a real-valued continuous function on $Y$. For each $\lambda \in \Lambda$, by the same argument as in $[3,8.20]$, there is $\gamma_{\lambda} \in W\left(\omega_{\alpha}\right)$ such that $\theta_{\lambda}=\phi \circ\left(h_{\lambda} \mid h_{\lambda}^{-1}(Y)\right)$ takes on the constant value $t_{\lambda}$ on $\left\{\left(\lambda(1), \gamma, \omega_{0}\right) \mid \gamma_{\lambda}\right.$ $\left.\leqslant \gamma<\omega_{\alpha}\right\} \cup\left\{\left(\lambda(2), \gamma, s_{\lambda}\right) \mid \gamma_{\lambda} \leqslant \gamma<\omega_{\alpha}\right\}$. Since

$$
\theta_{\lambda}\left(\left(\lambda(1), \omega_{\alpha}, \beta\right)\right)=\theta_{\mu}\left(\left(\mu(1), \omega_{\alpha}, \beta\right)\right)
$$

for $\lambda, \mu \in \Lambda$ and for each $\beta<\omega_{0}$, we have $t_{\lambda}=t_{\mu}$ for $\lambda, \mu \in \Lambda$. Extend $\phi$ over $Y_{0}$ by setting $\phi\left(y_{0}\right)=t_{\lambda}$. Then it is easy to see that the extension $\phi$ is 
continuous. Thus $Y$ is $C$-embedded in $Y_{0}$, and hence $Y_{0} \subset v Y$. It remains to show that $X \times Y$ is not $C$-embedded in $X \times v Y$. For each $\lambda \in \Lambda$ and each $\sigma \in \Sigma_{\lambda}$, let us set

$$
\begin{gathered}
y(\lambda, \sigma)=h_{\lambda}\left(\left(\lambda(2), \omega_{\alpha}, \sigma\right)\right), \\
K(\lambda, \sigma)=h_{\lambda}\left(\left\{(\lambda(2), \gamma, \sigma) \mid \gamma \leqslant \omega_{\alpha}\right\}\right) .
\end{gathered}
$$

And let us set

$$
\begin{aligned}
p(\lambda, \sigma) & =(x(\lambda, \sigma), y(\lambda, \sigma)) \in X \times Y, \\
L(\lambda, \sigma) & =H(\lambda, \sigma) \times K(\lambda, \sigma) \subset X \times Y, \\
\mathcal{L} & =\left\{L(\lambda, \sigma) \mid \lambda \in \Lambda, \sigma \in \Sigma_{\lambda}\right\} .
\end{aligned}
$$

Then $L(\lambda, \sigma)$ is a neighborhood at $p(\lambda, \sigma)$ in $X \times Y$. Now we show that $\mathcal{E}$ is discrete in $X \times Y$. To do this, let $p=(x, y) \in X \times Y$; then $y=$ $h_{\mu}((\mu(i), \delta, t))$ for some $\mu \in \Lambda, i \in\{1,2\}, \delta \leqslant \omega_{\alpha}$ and $t \in W\left(\omega_{0}+1\right) \oplus S_{\mu}$. If $t \in W\left(\omega_{0}+1\right)$ and $t<\omega_{0}$, then

$$
V(y)=\cup\left\{h_{\lambda}\left(T_{\lambda(1)}\right) \mid \lambda \in \Lambda\right\} \cap Y
$$

is a neighborhood at $y$ in $Y$ such that $V(y) \cap K(\lambda, \sigma)=\varnothing$ for each $\lambda \in \Lambda$ and each $\sigma \in \Sigma_{\lambda}$, and hence $X \times V(y)$ is a neighborhood at $p$ which meets no member of $\mathcal{L}$. If $t=\omega_{0}$ or $s_{\mu}$, then there exist $\tau \in \Sigma_{\mu}$ and a neighborhood $V(x)$ at $x$ such that $V(x) \cap G(\mu, \tau)=\varnothing$. If we set

$$
\begin{aligned}
V(y)= & h_{\mu}\left(\left\{(\mu(1), \gamma, \beta) \mid \gamma \leqslant \delta, \beta \leqslant \omega_{0}\right\}\right. \\
& \cup\{(\mu(2), \gamma, s) \mid \gamma \leqslant \delta, s \in J(\mu, \tau)\}),
\end{aligned}
$$

then $V(y)$ is a neighborhood at $y$ in $Y$ such that $V(x) \times V(y)$ meets no member of $\mathcal{L}$. If $t \in \Sigma_{\mu}$, then $X \times K(\mu, t)$ is a neighborhood at $p$ which meets only $L(\mu, t)$. Hence $\mathcal{E}$ is discrete in $X \times Y$. For each $\lambda \in \Lambda$ and each $\sigma \in \Sigma_{\lambda}$, there is a real-valued continuous function $\psi_{(\lambda, \sigma)}$ on $X \times Y$ such that $\psi_{(\lambda, \sigma)}(p(\lambda, \sigma))=0$ and $\psi_{(\lambda, \sigma)}(q)=1$ for each $q \in(X \times Y)-L(\lambda, \sigma)$. If we define a function $\psi$ by

$$
\psi(q)=\inf \left\{\psi_{(\lambda, \sigma)}(q) \mid \lambda \in \Lambda, \sigma \in \Sigma_{\lambda}\right\}, \quad q \in X \times Y,
$$

then $\psi$ is continuous, since $\mathcal{E}$ is discrete. For our purpose, it suffices to show that $\psi$ admits no continuous extension to the point $p_{0}=\left(x_{0}, y_{0}\right) \in X \times v Y$. Let $U$ be a given neighborhood at $p_{0}$. There exist $\mu \in \Lambda$ and a neighborhood $V\left(y_{0}\right)$ at $y_{0}$ in $Y_{0}$ such that $p_{0} \in G_{\mu} \times V\left(y_{0}\right) \subset U$. Then $y(\mu, \tau) \in V\left(y_{0}\right)$ for some $\tau \in \Sigma_{\mu}$, and hence $p(\mu, \tau) \in U$ and $\psi(p(\mu, \tau))=0$. On the other hand, $y(\beta)$ is in $V\left(y_{0}\right)$ for some $\beta<\omega_{0}$, and then $q=\left(x_{0}, y(\beta)\right) \in U$ and $\psi(q)=$ 1. This shows that $\psi$ does not extend continuously to $p_{0}$. Hence the proof is completed.

Before proving Theorem 2, we prove the implication (a) $\rightarrow$ (b) of Theorem 4 , which slightly improves a theorem of Comfort [1, Theorem 2.4]. We denote 
by $\mu X$ the topological completion of $X$ (i.e., the completion of $X$ with respect to its finest uniformity).

Proof of Theorem 4. (a) $\rightarrow$ (b). Assume that $v X$ is locally compact and card $X$ is nonmeasurable. Let $Y$ be a $k$-space. Then, by [1, Theorem 2.1], $v X \times Y$ is $C$-embedded in $v X \times v Y$. Since $v X$ is locally compact, by [5, Theorem 1.5], we have $v X=\mu X$. Hence $\mu(X \times Y)=\mu X \times \mu Y$ holds by [5, Theorem 2.3], and so $X \times Y$ is $C$-embedded in $\mu X \times Y(=v X \times Y)$. Thus we have $v(X \times Y)=v X \times v Y$.

Proof of TheOREM 2. (a) $\rightarrow$ (b). Let $X$ be a locally pseudocompact space of nonmeasurable cardinal and let $Y$ be a $k$-space. Now it suffices to show that for each pseudocompact subset $S$ of $X, S \times Y$ is $C$-embedded in $S \times v Y$. To see this, let $S$ be a given pseudocompact subset of $X$, then we have $v S=\beta S$ by [3, 8A4]. Thus $v(S \times Y)=v S \times v Y$ holds by Theorem 4, (a) $\rightarrow$ (b) proved above, and hence $S \times Y$ is $C$-embedded in $S \times v Y$. (b) $\rightarrow$ (a). Suppose on the contrary that $X$ is not locally pseudocompact at $x_{0} \in X$. Let $\left\{G_{\lambda} \mid \lambda \in \Lambda\right\}$ be a neighborhood base at $x_{0}$. Then, for each $\lambda \in \Lambda, \mathrm{cl}_{X} G_{\lambda}$ is not pseudocompact, and thus we can find a countable decreasing family $\left\{G(\lambda, \sigma) \mid \sigma \in \Sigma_{\lambda}\right\}$ of open sets in $X$ such that $\cap\left\{\operatorname{cl}_{X} G(\lambda, \sigma) \mid \sigma \in \Sigma_{\lambda}\right\}=\varnothing$ and $G(\lambda, \sigma) \subset G_{\lambda}$ for each $\sigma \in \Sigma_{\lambda}$. Let us set $H(\lambda, \sigma)=G(\lambda, \sigma)$, and choose a point $x(\lambda, \sigma) \in H(\lambda, \sigma)$. We construct $Y_{0}$ and $Y$ quite similarly to the proof of Theorem 1. Examining the process, one sees that then each $S_{\lambda}$ is compact, and hence $Z$ is locally compact. Since every quotient space and open subspace of a $k$-space is a $k$-space, $Y$ is a $k$-space. Therefore, by pursuing the proof of Theorem 1, we have Theorem 2.

To prove the implication (b) $\rightarrow$ (a) of Theorems 3 and 4, we need a theorem of Hušek [4, Theorem 3]. His theorem can be restated as follows:

HUŠEK's THEOREM. For a space $X$ the following conditions are equivalent:

(a) card $X$ is nonmeasurable.

(b) $v(X \times Y)=v X \times v Y$ holds for each discrete space $Y$.

Proof of Theorem 3. (a) $\rightarrow$ (b) is the result of Comfort quoted in the introduction. (b) $\rightarrow$ (a). By Hušek's theorem, card $X$ is nonmeasurable. It follows from Theorem 1 and [6, Theorem 5.2] that $X$ is locally compact and realcompact.

Proof of Theorem 4. (b) $\rightarrow$ (a). Since a discrete space is a $k$-space, by Husek's Theorem, card $X$ is nonmeasurable. By Theorem $2, v X$ is locally pseudocompact, and hence is locally compact, because every pseudocompact realcompact space is compact (cf. [3, 8E1]).

3. Remarks. (1) If $v X$ is locally compact, then $X$ is locally pseudocompact, but the converse is false (see [1]).

(2) The space $Y$ constructed in the proof of Theorems 1 and 2 and [6, Theorem 5.2] is 0-dimensional (i.e., ind $Y=0$ ). Hence all theorems in this note remain true if "for each $(k-)$ space $Y$ " is replaced by "for each 0-dimensional $(k$-) space $Y$ ". 
(3) A space similar to the space $S_{\lambda}$ in the proof of Theorem 1 was used in [6] to show that every member of $R$ is realcompact.

(4) A space $X$ is said to be topologically complete if it is complete with respect to its finest uniformity (i.e., $X=\mu X$ ). In [7], Morita proved that if $X$ is locally compact topologically complete, then $\mu(X \times Y)=\mu X \times \mu Y$ holds for each space $Y$, and Isiwata [5] proved that if $\mu(X \times Y)=\mu X \times \mu Y$ holds for each space $Y$, then $X$ is topologically complete (cf. also [8]). Hence the analogous results of Theorems $1 \sim 4$ remain true, with no cardinality conditions, for topological completions (in this case, we need to use [5, Theorem 2.3], [7, Theorem 3.1] and [2, Lemma 3.1] instead of Theorem 4, (a) $\rightarrow$ (b), [3, 8A4] and [3,8E1], respectively).

ADDED IN Proof. Recently, Blair and Hager (z-embedding in $\beta X \times \beta Y$, Set theoretic topology, Academic Press, New York, 1977) asked whether the following condition ( $\mathrm{d}^{\prime}$ ) implies that $X \times Y$ is $z$-embedded in $\beta X \times \beta Y$ (i.e., each zero-set of $X \times Y$ is the trace on $X \times Y$ of a zero-set of $\beta X \times \beta Y$ ):

(d') For every real-valued continuous function $f$ on $X \times Y$ and every $\varepsilon>0$, there is a countable open rectangular cover $\left\{G_{n}\right\}$ of $X \times Y$ such that $\sup \left\{|f(p)-f(q)| \mid p, q \in G_{n}\right\}<\varepsilon$ for each $n$.

In the same paper, they proved that if $X$ has a countable base, then $X \times Y$ satisfies $\left(\mathrm{d}^{\prime}\right)$ for each space $Y$, and that if $X \times Y$ is $z$-embedded in $\beta X \times \beta Y$, then $v(X \times Y)=v X \times v Y$ holds. From these facts, since there exists a nonlocally compact space with a countable base, Theorem 3 answers this question negatively. Furthermore, combining Theorem 3 with their results (3.2, 3.3), we obtain: $X$ is a locally compact space with a countable base if and only if $X \times Y$ is $z$-embedded in $\beta X \times \beta Y$ for each space $Y$.

\section{REFERENCES}

1. W. W. Comfort, On the Hewitt realcompactification of a product space, Trans. Amer. Math. Soc. 131 (1968), 107-118.

2. N. Dykes, Mappings and realcompact spaces, Pacific J. Math. 31 (1969), 347-358.

3. L. Gillman and M. Jerison, Rings of continuous functions, Van Nostrand, Princeton, N. J., 1960.

4. M. Hušek, Pseudo-m-compactness and $v(P \times Q)$, Indag. Math. 33 (1971), 320-326.

5. T. Isiwata, Topological completions and realcompactifications, Proc. Japan Acad. 47 (1971), 941-946.

6. W. G. McArthur, Hewitt realcompactifications of products, Canad. J. Math. 22 (1970), 645-656.

7. K. Morita, Topological completions and M-spaces, Sci. Rep. Tokyo Kyoiku Daigaku Sect. A 10 (1970), 271-288.

8. R. Pupier, Topological completion of a product, Rev. Roumaine Math. Pures Appl. 19 (1974), 925-933.

Department of Mathematics, University of Tsukuba, Sakuramura Ibaraki, 300-31, JAPAN 